\title{
ESTRATEGIAS DE SUPERVIVENCIA: UN CONCEPTO EN BUSCA DE SU CONTENIDO
}

\author{
OMAR ARGÜELLO \\ CELADE, Santiago, Chile
}

\section{INTRODUCCIÓN}

EN EL MUNDO de los científicos sociales latinoamericanos un pequeño fantasma recorre sus encuentros, seminarios, reuniones de trabajo, talleres, etc. Se lo intenta aprehender con la expresión "estrategias de supervivencia". Si se quieren estudiar los problemas de los campesinos, nada mejor que plantearse un proyecto sobre sus "estrategias de supervivencia"; lo mismo vale para los trabajadores del agro, los marginales de la ciudad e incluso para grupos sociales que tienen problemas muy diferentes de los anteriores, como pueden ser los diversos grupos y capas de la burguesía.

Como este concepto ha sido difundido en torno al Programa de Investigaciones sobre Población en América Latina (PISPAL), el mismo ha pasado a recubrir toda la problemática poblacional. Si se quiere saber algo sobre las migraciones será altamente conveniente plantearse el tema desde la perspectiva que construye ese objeto de estudio como parte del conjunto de acciones y decisiones que dan lugar a una estrategia de supervivencia. Si se quiere estudiar la fecundidad, no parece existir alternativa más adecuada que plantearse el tema como reproducción de la población o de la fuerza de trabajo, tanto en sus aspectos materiales como biológicos, dentro de una estrategia como la ya mencionada. Suponemos que la mortalidad, cuando se la estudie más sistemáticamente, también se la planteará dentro de la perspectiva de las estrategias (¿frustradas?) de supervivencia. ${ }^{1}$

Frente a esta diversidad temática y a la ambigüedad respecto a los aspectos de la realidad social que se quieren aprehender y analizar, se hace necesario circunscribir mejor el fenómeno social que se busca conceptualizar, así como resolver varios problemas teóricos y metodológicos que dificultan esa conceptualización. Con esto se intenta reorientar un procedimiento extraño

' El autor recoge esta impresión a través de su participación en las siguientes reuniones recientes: Taller sobre Estrategias de Supervivencia, organizado por PISPAL y realizado en Buenos Aires, Argentina (CEUR) en marzo de 1980; Reunión de Trabajo del Grupo de Migraciones de la Comisión de Población y Desarrollo de CLACSO, celebrada en Buenos Aires, Argentina (CENEP), en agosto de 1980; y Seminario sobre Estructura Agraria y Población, organizado por PIS,PAL y realizado en Sao Paulo, Brasil (CEBRAP) en septiembre de 1980. 
e irregular que ha tenido lugar respecto del fenómeno de las estrategias de supervivencia. A nuestro juicio la práctica científica llega a la elaboración de conceptos como resultado de una reflexión en torno a problemas específicos o como respuestas a preguntas que se hacen frente a aspectos parciales de la realidad social. Con el concepto de "estrategias de supervivencia" parece estar ocurriendo lo contrario: frente a una rápida y generosa difusión de este preconcepto, se busca utilizarlo para cubrir nuevas y diferentes áreas de la realidad social, asignándole cada vez nuevos contenidos y haciendo peligrar con ello la identificación y tratamiento de un fenómeno específico que afecta a un grupo determinado de la sociedad y que plantea un tema científico y socialmente relevante.

Con estas notas se pretende reivindicar el lugar que este concepto de "estrategias de supervivencia" tenia dentro del discurso de las ciencias sociales; para ello se hace necesario volver a preguntarse cuál es el fenómeno social o el aspecto de esa realidad que se quiere aprehender, único camino para llegar a elaborar un concepto adecuado y para diferenciarlo de otros conceptos que apuntan a fenómenos sociales diferentes. En ese camino, comenzaremos por 'revisar los contenidos de los conceptos de "estrategias familiares de vida" y de "estrategias de supervivencia" para diferenciarlos, enfatizando la distinción entre "reproducción biológica" y "reproducción material de la población"; en un punto posterior discutiremos qué grupo o grupos sociales se ven enfrentados a situaciones sociales y económicas que los obligan a ensayar diferentes estrategias de supervivencia; finalmente, se propondrá una definición provisoria y se dejará anotado un conjunto de problemas teóricos y metodológicos que deberán resolverse dentro de la investigación sobre el tema (entre otros, las relaciones posibles entre estas estrategias y la investigación socio-demográfica).

\section{ESTRATEgIAS DE SUPERVIVENCIA Y ESTRATEGIAS FAMILIARES DE VIDA: DIFERENCIAS DE CONTENIDO}

La expresión "estrategias de supervivencia" nace, hasta donde conocemos, en un trabajo de Duque y Pastrana, quienes se plantearon analizar las formas en que lograban sobrevivir las familias de pobladores de dos campamentos ${ }^{2}$ del Gran Santiago en Chile. Los autores se preocupan de la supervivencia económica de estas familias y no dan ningún papel particular a la reproducción biológica dentro del fenómeno social que están interesados en estudiar. Dichos autores hablan de "estrategia objetiva de subsistencia económica" y afirman que "el aspecto central de ella consiste en la reordenación de funciones al interior de las unidades familiares, enfatizando la participación económica de todos o la mayoría de los miembros componentes: la madre, los hijos mayores y

2 Duque, J. y Pastrana, E., Las Estrategias de Supervivencia Económica de las Unidades Familiares del Sector Popular Urbano: Una Investigación Exploratoria. Programa ELAS/CELADE, Santiago, Chile, 1973. Los autores aclaran que el campamento es una modalidad de poblamiento espacial formado por un conjunto de familias sin casa, quienes "toman y se apropian" de predios urbanos. p. 42 
menores, los allegados y aun los miembros consanguíneos próximos". ${ }^{3}$

Ratificando el contexto económico que definen las estrategias de supervivencia, los mismos autores vuelven sobre lo mismo en otras partes de su obra, sosteniendo que "las estrategias de supervivencia económica familiar consisten principalmente en la acentuación en el ejercicio de funciones económicas para todos o la mayoría de los miembros constituyentes de las unidades familiares... Para la estrategia de supervivencia económica los componentes familiares comienzan a funcionar como un ejército de reserva, movilizable según las diversas contingencias que afectan al fondo familiar de consumo". ${ }^{4} \mathrm{Si}$ bien los hijos mayores y menores cumplen un papel en la obtención del ingreso familiar, junto con otros parientes y extrafamiliares, nunca aparece en los autores citados, una referencia que permita atribuir a la reproducción biológica un papel importante dentro de su definición de "estrategias de supervivencia". Sólo se interesan por la supervivencia económica, o si se quiere, de la reproducción material de los miembros de la unidad familiar.

El contenido del concepto va a sufrir un cambio brusco con la definición que del mismo replantea PISPAL al fijar sus líneas prioritarias varios años después. En el nuevo contenido que PISPAL atribuye a las "estrategias de su-. pervivencia" se habla ahora de comportamientos encaminados a asegurar la reproducción material y biológica del grupo, poniendo como ejemplo de las mismas, la participación económica, para el caso de la reproducción material (al igual que Duque y Pastrana) y agregando ahora una serie de comportamientos demográficos como formación y disolución de las uniones, que le son necesarios para poder introducir la reproducción biológica. ${ }^{5}$

Volviendo ahora sobre el tema, Torrado, quien había tenido activa participación en la definición elaborada por PISPAL en 1978, ${ }^{6}$ hace una nueva enumeración de los comportamientos básicos que estarían incluidos en las estrategias para el caso de la clase obrera que ella se propone estudiar. Estos son: a) constitución de la unidad familiar; b) procreación; c) preservación de la vida; d) socialización y aprendizaje; e) ciclo de vida familiar; f) división familiar del trabajo; g) organización del consumo familiar; h) migraciones laborales; i) localización residencial; j) allegamiento cohabitacional; k) cooperación extrafamiliar. Debe tenerse en cuenta que cada uno de estos

\footnotetext{
${ }^{3}$ Duque, J.y Pastrana, E., op. cit., p. 177. Merecen destacarse dos aspectos de esta primera definición de estrategias de supervivencia hecha por los autores: a) ellos hablan de estrategia "objetiva", lo que parece indicar que no se requiere ninguna conciencia por parte de los agentes que desarrollan la estrategia; b) pese a hablarse de estrategias familiares, en realidad se tiene en mente la unidad doméstica, pues se habla de la participación económica de los "allegados" quienes no son necesariamente familiares, lo que se ratifica en $p .178$ cuando se dice que en el ingreso familiar subproletario también participan "otros miembros extra-familiares".

Duque, J., y Pastrana, E., op. cit., p. 21

PISPAL, Líneas Prioritarias de Investigación para la 111 Fase. México, Agosto, 1978, p. 7-8

- Torrado, S., Sobre los conceptos de "estrategias familiares de vida" y "proceso de reproducción de la fuerza de trabajo": notas teórico-metodológicas. CEUR, Argentina, 1980. Ver especialmente nota cuatro en página tres.
} 
enunciados es seguido de un listado de comportamientos relacionados con el punto, por lo cual estos enunciados deben tomarse más bien como dimensiones alrededor de las cuales pueden nuclearse otra serie de comportamientos básicos. Nótese además que se engloban nuevamente comportamientos claramente dirigidos a la reproducción biológica, junto con otros claramente dirigidos a la reproducción material.

Frente a esta diversidad de dimensiones del comportamiento social de una familia, Torrado no podia ignorar que se encontraba en realidad frente a un nuevo fenómeno social y que el contenido derivado del mismo ya no era concordante con el concepto de "estrategias de supervivencia"; por lo cual propone, acertadamente, un nuevo concepto adecuado al tipo de fenómeno social que estaba relevando, y así nacen las "estrategias familiares de vida". El contenido de este nuevo concepto se diferencia del correspondiente al de "estrategias de supervivencia" en dos aspectos fundamentales: a) a la preocupación por la reproducción material de la población a través de su participación económica, se agregan ahora los comportamientos encaminados a asegurar la reproducción biológica del grupo; b) de un fenómeno social que sólo alcanzaba a ciertos grupos subordinados y explotados, ahora se trata de un fenómeno social que alcanza a todos los grupos y clases sociales. Por ello la autora dice que este fenómeno constituye "un aspecto fundamental de la dinámica de reproducción de las diferentes clases y estratos sociales de una sociedad concreta".?

No hay dudas que el nuevo concepto de "estrategias familiares de vida" hace referencia a un fenómeno social más global y más rico en dimensiones dentro de su contenido, comparado con las "estrategias de supervivencia"; tampoco hay duda de que estas últimas son una parte de aquellas estrategias de vida para algunos grupos determinados dentro del conjunto de la sociedad. Esto no debe significar, sin embargo, que la aparición de un nuevo concepto más general, sustituya o borre del discurso científico a los conceptos más especificos que recortan aspectos parciales de la realidad social que hacen parte de aquel fenómeno más global. De lo contrario, debiéramos arrojar por la borda conceptos como los de socialización y aprendizaje; migraciones laborales; o división familiar del trabajo, que también hace parte de los comportamientos básicos enumerados por Torrado dentro de los componentes de las "estrategias familiares de vida". Lo mismo es plenamente válido para las "estrategias de supervivencia", las que, al igual que los anteriores, ostentan suficiente autonomía relativa como para justificar un desarrollo conceptual propio que le permita su tratamiento científico a nivel teórico y empírico.

Cabria preguntarse por qué PISPAL, en 1978, al fijar sus líneas prioritarias da un vuelco en torno a las "estrategias de supervivencia" y amplía su contenido al preocuparse tanto de la reproducción material como de la biológica. No es tarea nuestra resolver este problema, pero pensamos que la preocupación poblacional de este programa puede haber influido al sentir la necesidad de incorporar nuevos componentes demográficos a los com-

\footnotetext{
${ }^{7}$ Torrado, S., op. cit., p. 3.
} 
portamientos básicos de aquella estrategia. Por eso aparecerán los componentes de constitución de las uniones y procreación, entre otros. Junto con esto, y quizás más importante que lo anterior, debe haber influido una perspectiva que tiende a vincular siempre los fenómenos de reproducción de la población con aquéllos de la reproducción de la fuerza de trabajo. Esta perspectiva tiende a observar el proceso a partir de las necesidades del capital y por lo tanto se preocupará tanto de la reproducción material de su fuerza de trabajo como de la reproducción biológica de la misma, en este proceso de mediano o largo plazo.

Esto que también ocurre con las "estrategias familiares de vida", no es el caso de los fenómenos de la supervivencia, donde sólo se hace referencia a los arreglos o mecanismos necesarios para la reproducción material de ciertos subgrupos de población. No se trata tampoco de negar a priori que en. algunos casos la procreación pueda hacer parte de la estrategia de supervivencia. Cuando ésta tenga como unidad de acción a la familia, como es el caso más frecuente aunque no el único, ${ }^{8}$ es posible que algunas de estas familias recurran a la procreación como medio de obtener, a mediano plazo, una ayuda en su supervivencia. Pero en estos casos se trataría de un mecanismo o comportamiento básico más, dentro de los muchos y variados a los que recurren los que se encuentran en situación de pelear por su supervivencia. En estos casos, la procreación será otro de los "medios" a los que se recurriría para lograr el "fin" de sobrevivir o reproducirse materialmente. Pero la procreación o reproducción biológica nunca entra como un fin dentro de las estrategias de supervivencia, como lo es para la reproducción de la fuerza de trabajo o dentro de la reproducción de la población.

Vimos entonces, aunque someramente, los contenidos (referentes empíricos, áreas temáticas o aspectos parciales de la realidad social que son recortados) de los conceptos de "estrategias familiares de vida" y de "reproducción de la fuerza de trabajo". Para contrastarlos y diferenciarlos del contenido que encierra el concepto de "estrategias de supervivencia" debemos resumir aquí brevemente las ideas que han estado apareciendo en páginas anteriores respecto de este último concepto. En síntesis podemos decir que el tipo de fenómeno social que relevaron Duque y Pastrana hace ya varios años sigue totalmente vigente y por lo tanto debe volver a ser el que da sentido y contenido al concepto de "estrategias de supervivencia". Se trata de los arreglos, mecanismos o comportamientos específicos que debe ensayar un sector determinado de la población de las sociedades nacionales latinoamericanas, tendientes a lograr su reproducción material, dada la incapacidad mostrada por el sistema productivo para asegurarles una actividad económica estable de la cual se deriven los ingresos necesarios para ello. Como vimos también, y para diferenciarlo del contenido de las "estrategias familiares de vida", no se trata de comportamientos que se preocupen de la reproducción biológica del grupo, no se refiere a todos los grupos y clases de

${ }^{8}$ Ver nota 2 de la p. 3, donde se destaca como Duque y Pastrana hablan del aporte de miembros extrafamiliares de la unidad doméstica para posibilitar la supervivencia. 
la sociedad concreta, y no siempre la supervivencia se organiza a través de la unidad familiar, aun cuando pueda ser el caso más frecuente.

Dadas estas diferencias fundamentales en el contenido de ambos conceptos, $\mathrm{y}$ dado el hecho que ambos utilizan además expresiones distintas para su enunciado, es de esperar que ya no se utilicen más como sinónimos, ni como sustituibles, los conceptos de "estrategias de supervivencia" y de "estrategias familiares de vida".

\section{SUPERVIVENCIA DE QUIEN?}

No han faltado trabajos que hablen de estrategias de supervivencia refiriéndose a ciertos grupos definidos fundamentalmente por sus características culturales más que por sus características socioeconómicas. Es el caso de estudios como el referido a migrantes de las Indias Occidentales residentes en Los Angeles, Estados Unidos de América, donde se analizan formas de adaptación y organización social de estos migrantes para preservar su identidad cultural y defenderse de discriminaciones de la sociedad receptora. ${ }^{9}$ También ha llegado a hablarse de "supervivencia" en el diálogo entre naciones, como es el caso del Informe de la Comisión Brandt sobre relaciones norte-sur para lograr la supervivencia de la comunidad internacional. ${ }^{10}$

Sin embargo, parece existir un cierto consenso fáctico entre los investigadores latinoamericanos respecto a que el concepto de "estrategias de supervivencia" apunta a la aprehensión, comprensión y explicación de un determinado comportamiento de ciertos grupos subordinados que no logran una inserción estable en la estructura productiva nacional. En la convocatoria hecha por PISPAL para el Taller sobre "estrategias de supervivencia" se habla de diferencias considerables en las mismas según se consideren unidades familiares rurales o urbanas, aun cuando comparten ciertas características de tipo estructural, como su similar nivel de pobreza. "Duque y Pastrana ${ }^{12}$ comenzaron a usar este concepto en 1973, y lo hicieron al estudiar poblaciones marginales urbanas. Son estos mismos grupos marginales los que considera Lomnitz ${ }^{13}$ al estudiar las estrategias de supervivencia en barriadas de México; otros, en general, se refieren a este tema tomando siempre a grupos o sectores populares.

Parece entonces que de hecho y particularmente al nivel de trabajos empiricos, las estrategias de supervivencia son referidas sóto a determinados grupos socioeconómicos. Es cierto que todos los individuos, todas las familias,

" Joyce Bennett Justus, "Strategies for survival: West Indians in Los Angeles", en H. E. Lamur y J. D. Speckmann (Eds.). Adaptation of Migrants from the Caribbean in the European and American Metropolis. Co-publication of the University of Ambsterdam and the Royal Institute of Linguistics and Anthropology at Leiden, Netherlands, 1975.

${ }^{10}$ North-South: A programme for survival. The Report of the Independent Commission on International Development Issues under the Chairmanship of Willy Brandt. Pan Books Ltd., 1980.

${ }_{12}^{1}$ El subrayado es nuestro.

12 Duque y Pastrana, op. cit.

13 Lomnitz L.A. de. Cómo sobreviven los marginados. Siglo XXI, México, 1975. 
de todos los grupos y clases sociales sobreviven determinados períodos de tiempo, reproduciéndose materialmente a través de diversos comportamientos. Pero, a nuestro juicio, existe un subconjunto de comportamientos específicos que debe ensayar un grupo de la población nacional, que no es comparable con los comportamientos que ensayan otros grupos sociales, y que por lo tanto dan lugar a un fenómeno nuevo propio de estos grupos y no generalizables al conjunto de la población.

Estos grupos deberán ser determinados en cada sociedad concreta a través de los trabajos de investigación teórica y empírica que se hagan sobre el tema. Para ubicarlos en términos de clases o fracciones de clases sociales, podemos adelantar que se tratará en general de elementos de la pequeña burguesía urbana y rural (pequeños artesanos y minifundistas) con medios de producción insuficientes para sobrevivir, lo cual los lleva a recurrir a mecanismos heterodoxos respecto de su posición de clase, como venta temporaria de su fuerza de trabajo, uso del trabajo de los hijos menores para completar el ingreso, etc.; también se tratará de elementos dentro del proletariado, del subproletariado y de integrantes del ejército industrial de reserva, a quienes el modo de producción capitalista dependiente, predominante en nuestras sociedades, no da oportunidad de vender su fuerza de trabajo en forma estable para actividades económicas suficientemente productivas, lo que los lleva también a mecanismos heterodoxos a su posición de clase, como diversas formas de trabajo por cuenta propia que disfrazan sus situaciones de desempleo o subempleo, además del recurso frecuente del trabajo de sus hijos menores.

\section{IV: UNA DEFINICIÓN POSIBLE Y PROBLEMAS PENDIENTES}

De acuerdo con las especificidades anotadas en los puntos anteriores, proponemos definir provisoriamente las "estrategias de supervivencia" como el conjunto de acciones económicas, sociales, culturales y demográficas que realizan los estratos poblacionales que no poseen medios de producción suficientes ni se incorporan plenamente al mercado de trabajo, por lo que no obtienen de las mismas sus ingresos regulares para mantener su existencia en el nivel socialmente determinado, dadas las insuficiencias estructurales del estilo de desarrollo predominante.

Esta definición, aún provisoria, requiere de mayores especificaciones relativas a varios aspectos derivados directa o indirectamente de la misma. En principio pueden anotarse los siguientes aspectos que merecen una amplia discusión y esclarecimiento metodológico:

a) Cómo determinar los grupos o estratos que en una sociead concreta están obligados al desarrollo de estrategias de supervivencia o cuáles aparecen como relevantes para ser investigados dentro de esta preocupación temática, y cómo aprehenderlos empiricamente;

b) ćomo determinar las diversas dimensiones que componen la estrategia 
de supervivencia, y cómo establecer cuáles acciones del cotidiano de las unidades grupales hacen parte de dichas estrategias, cómo jerarquizar y sistematizar dichas acciones y cómo determinar que apuntan o no a la satisfacción de las necesidades básicas;

c) cómo conceptualizar los "estilos de desarrollo", y cómo operacionalizarlos para diferenciar la posible diversidad de ellos en la región latinoamericana, y para asociar empíricamente determinados estilos cie desarrollo a diversas estrategias de sobrevivencia;

d) definición de la unidad de análisis, y

e) establecer las técnicas de recolección de información capaces de aprehender lo cotidiano de la estrategia de supervivencia, pero con una cobertura espacial mayor que la pequeña comunidad.

Sin pretender resolver tantos y tan complejos problemas, se hacen aquí algunos comentarios que amplían los puntos anotados anteriormente.

a) Determinación de los agentes o grupos sociales que deben ensayar estrategias de supervivencia:La definición provisoria propuesta indica las situaciones estructurales que llevarán a los agentes sociales a ensayar las referidas estrategias (medios de producción rurales o urbanos insuficientes y una incorporación no plena al mercado de trabajo urbano o rural) pero no especifica ninguna categoría específica en términos de clases o fracciones de clase social. Podríamos hablar del campesinado en descomposición o de la pequeña burguesía urbana y rural pauperizada con medios insuficientes de producción, así como de fracciones de la clase obrera y componentes del ejército industrial de reserva que no logran incorporarse plenamente al mercado de trabajo organizado alrededor del sector más dinámico dentro del modo de producción capitalista dependiente. Pero esto no resuelve todo el problema ni suple la necesidad de desarrollos metodológicos que permitan la identificación de los observables en la investigación empírica.

Sabemos ya que hasta ahora los trabajos sobre el tema se han referido a "sectores populares", "grupos marginales", "campesinos pobres", etc. Pero se hace necesaria una elaboración teórica metodológica que legitime científicamente esas elecciones fácticas; aquí sólo se mencionarán algunos criterios básicos para esa tarea, postulándose que la forma más adecuada para esos avances estaría dada por la práctica científica en investigaciones concretas. El criterio básico para la identificación buscada es el tipo y grado de inserción de los agentes en la estructura productiva. Las capas de la pequeña burguesía urbana y rural y las fracciones de clase proletaria y ejército de reserva mencionadas anteriormente deberán ser identificadas en las fuentes de información respecto a la rama de actividad y grupos ocupacionales, tamaño y características de las pequeñas empresas agrícolas y urbanas, así como el grado de desempleo y subempleo visible; también se necesitará conocer otras formas de subempleo invisible como ha propuesto la Organización Internacional del Trabajo, entre ellas cuando la productividad real está por debajo de la productividad "potencial" utilizándose generalmente, por 
falta de información, los niveles de ingreso medio como variable proxy de la productividad..$^{14}$

Lamentablemente, en los proyectos de investigación sobre el tema se descuidan estas elaboraciones teórico-metodológicas y se recurre a otros criterios, como zonas de residencia, generalmente barrios marginales dentro de las grandes ciudades o áreas de minifundios agrarios. Sin excluir este tipo de decisiones operacionales, válidos particularmente para estudios de casos, deben crearse las condiciones para aprehender este fenómeno social en los diversos sectores y áreas de la realidad nacional. Esto se hace necesario cuando se está interesado en conocer la magnitud o proporción de la población que debe sobrevivir mediante estrategias particulares dentro de una sociedad nacional concreta. También es importante poder comparar los comportamientos básicos de diferentes grupos enfrentados a estrategias de supervivencia pero ubicados en diferentes contextos estructurales o de residencia, entre otros, contextos agrícolas versus contextos urbanos, o barrios básicamente marginales versus barrios heterogéneos en cuanto a niveles de pobreza o marginalidad (ifuncionan igualmente en todos los casos las redes de intercambio, la reciprocidad favorecida por la cercanía física, el cuidado reciproco de los hijos, y otros mecanismos como los detectados por Lomnitz?). ${ }^{15}$

b) Cómo determinar las diferentes dimensiones que componen las estrategias de supervivencia, y cómo establecer cuáles acciones concretas hacen parte de la misma, su sistematización y jerarquización, es otra tarea metodológica de importancia. El conjunto de acciones y comportamientos susceptibles de ser considerados parte de una estrategia de supervivencia, debe ser sistematizado en algunas dimensiones relevantes y para ello, en principio, puede pensarse en las tradicionales instancias de lo económico, lo social, lo cultural y lo demográfico.

i) En lo económico estarán presentes aquellas actividades que se proponen obtener un ingreso monetario, en especie o en forma susceptible de ser utilizado para la supervivenciä. No es necesario repetir aquí las catego-: rías económicas de ocupación, condición de actividad, categoría ocupacional, rama de actividad, sector informal, estabilidad en el empleo, subempleo visible e invisible, baja productividad, niveles de ingresos, formas de ingreso, etc. Sí es necesario destacar la urgencia de ajustar las técnicas de recolección de información sobre esas categorías económicas, ya que las mismas adolecen de muchas insuficiencias, particularmente en la medición del desempleo, subempleo e ingresos, lo que se agrava notablemente en cuanto a la capacidad de captar el aporte de los menores a la supervivencia del grupo;

ii) en lo social, interesa particularmente aprehender y analizar aquellas acciones que dan lugar a procesos particulares de organización social,

${ }_{14}$ PREALC, OIT, El Problema del Empleo en América Latina: Situación, Perspectivas y Políticas. Impreso en $P R E A L C$, Santiago de Chile, 1976, p. 11.

is Lomnitz, L.A. de. op. cit. 
como son las redes de intercambio, el rol de las mujeres y de los hijos en la obtención de los recursos del grupo, la organización para construcción de viviendas y para atención de mínimos servicios y de ayuda mutua;

iii) en lo cultural se hace necesario volver a discutir el concepto de "cultura de la pobreza". Los que han utilizado el mismo han caído, consciente o inconscientemente, en el sesgo de atribuir a estos aspectos culturales las causas de la pobreza y de las malas condiciones de existencia. Sin embargo, eso no debe llevarnos a un rechazo a priori de este concepto, el que puede ser de enorme utilidad en la investigación empírica como fenómeno mediatizador de las condiciones estructurales y de los comportamientos específicos de los individuos o grupos, así como de mucha utilidad en el diseño de políticas, ya que el conocimiento de esos hábitos y comportamientos es importante para decidir las acciones adecuadas. Parece difícil desconocer la existencia de hábitos, actitudes y comportamientos de grupos marginales, pobres o de precarias condiciones de existencia, que refuerzan los condicionantes estructurales y obstaculizan la posibilidad de ruptura de las situaciones de supervivencia. No hay duda de que estos hábitos, actitudes y comportamientos se originan y son causados por las condiciones estructurales del estilo de desarrollo imperante, por lo que en definitiva las raíces del fenómeno, las explicaciones causales y las variables independientes deben buscarse en esos condicionamientos estructurales. También es cierto que para superar esas condiciones de existencia será necesario remover esos condicionantes estructurales antes que cambiar las pautas características de la cultura de la pobreza.

Pero también parece cierto que la conceptualización y operacionalización de esta cultura de la pobreza ayudará a comprender la manifestación específica de aquellos condicionantes estructurales en diferentes grupos de población, como respuestas particulares a las características de diferentes estilos de desarrollo. Y el conocimiento de esas particularidades es esencial para la forma que adoptará una política de cambios sociales, ya que la misma apunta a medidas de fondo y a procedimientos de forma que hacen viables aquellas medidas;

iv) en lo demográfico se hacen necesarias algunas especificaciones mayores dada la vinculación estrecha que se viene haciendo del concepto de "estrategias de supervivencia" con la investigación sociodemográfica. A nuestro juicio existen por lo menos dos formas de vinculación de los temas demográficos con las mencionadas estrategias: 1) por un lado están las acciones o comporti mientos dèmográficos que hacen parte de la estrategia de supervivencia, independientemente del hecho de no ser la reproducción biológica de la población un objetivo de las mismas; 2 ) por otro lado, una serie de efectos demográficos se derivan de las mismas situaciones estructurales que obligan a ensayar estrategias particulares para su subsistencia a los grupos afectados. Dado el origen común de ambos fenómenos y la posibilidad de efectos propios de la interacción entre esos fenómenos demográficos y estratégicos, pensamos que se pre- 
senta aquí un nuevo campo para la investigación sociodemográfica. Como ejemplos del primer campo de investigación tenemos todo lo relacionado con la demografía económica, que comprenderá todos los aspectos referidos a las pautas de participación económica de los sectores que deben ensayar estrategias de supervivencia, incluyéndose entre los mismos a los movimientos migratorios laborales. También deberá investigarse, en los casos en que la unidad de acción de la estrategia es la familia, si alguna de ellas utiliza la procreación como un "medio" para ayudar a la obtención de los ingresos necesarios para la reproducción material de sus miembros.

Como ejemplos del segundo campo de investigación demográfica, tenemos los estudios de los posibles efectos que sobre la nupcialidad, fecundidad, mortalidad y migraciones, tienen las situaciones socioeconómicas: que llevan, a los afectados, a ensayar estrategias de supervivencia. Generalmente, los grupos poblacionales en esas situaciones estructurales tenderán a tener bajos ingresos, bajos niveles educacionales y ocupaciones menos estables, asociado todo ello a una nupcialidad más temprana, a un mayor número promedio de hijos, a una mortalidad promedio mayor, y son, además, los más expuestos a las necesidades de migrar dada su búsqueda por lograr una ubicación en la estructura productiva.

Estos efectos de la situación estructural sobre las dimensiones demográficas tendrán seguramente consecuencias sobre las estrategias de supervivencia, ya que muy posiblemente una nupcialidad más o menos temprana o un nủmero mayor o menor de hijos, obligará y permitirá comportamientos diversos. No faltarán quienes quieran ver estas pautas de nupcialidäd y de fecundidad como una acción que hace parte de una estrategia, consciente o no, para la supervivencia. Nosotros nos inclinamos por explicarlas como consecuencias de las situaciones estructurales mencionadas, dados los niveles de ingresos y de educación resultantes de las mismas; una vez que estas pautas de nupcialidad y de fecundidad se han concretado, son un hecho que deberá manipularse dentro de las estrategias de supervivencia, sin negar que aquéllas influirán necesariamente sobre éstas.

En todo caso, determinar si son acciones buscadas dentro de la estrategia, o si son hechos demográficos derivados de situaciones estructurales que deberán influir en las características de la estrategia de supervivencia, es un asunto que debe dejarse a la investigación empírica sobre el tema. El campo está delimitadó y sus componentes especificados; la dirección de la causalidad o de los condicionamientos debe buscarse en el análisis científico de la realidad social cuestionada. Lo que a nuestro juicio siempre queda claro es que estos comportamientos demográficos no son el objetivo de las estrategias de supervivencia; puede discutirse si se trata de medios utilizados para conseguir aquél objetivo de la reproducción material de los miembros del grupo, o si se trata de efectos de situaciones estructurales que afectan las características de esas estrategias de supervivencia; pero nunca se plantean como objetivos en sí mismos de estas estrategias. 
Finalmente, queremos sostener la independencia relativa de la investigación sociodemográfica respecto de las estrategias de supervivencia. La investigación cuyo objeto de estudio sean estas últimas estrategias, tendrá seguramente como parte de la misma a diversos componentes demográficos, aun cuando su objetivo trascienda los parámetros dados por estos componentes. Por otro lado, el estudio de los diversos fenómenos poblacionales, tomados en sus dimensiones aisladas o como sistema demográfico, ganará seguramente en claridad y en sus posibilidades explicativas, cuando logre aprehender a los mismos como parte integrada de estrategias que vinculen estos comportamientos demográficos con objetivos socioeconómicos particulares.

Pero debe quedar claro, también, que no siempre será necesario y pertinente vincular la dinámica demográfica a las estrategias de supervivencia, lo que indica la existencia de un campo de investigación sociodemográfica autónomo de la misma. Esto no empobrece en absoluto el tratamiento de lo demográfico, pues todavía sigue vigente el tratamiento teórico, metodológico y empírico del mismo, en sus relaciones con las situaciones estructurales y a través de las especificaciones por grupos y clases sociales. A veces, al contrario, este campo autónomo de la investigación sociodemográfica podrá avanzar mejor al no verse obstaculizada por algunas de las tareas teóricas y metodológicas pendientes en el trabajo con las "estrategias de supervivencia".

c) La conceptualización adecuada de los "estilos de desarrollo". Hace años se viene haciendo presente este término en los escritos de instituciones e investigadores de la región, sin que quede claro si esto sustituye y en qué medida perfecciona las nociones de estrategias o modelos de desarrollo, sistemas, etc. Aníbal Pinto ${ }^{16}$ reserva el término "sistema" para diferenciar el capitalismo del socialismo y el término "estructura" para diferenciar entre sociedades industrializadas y subdesarrolladas. Dentro de cada una de esas situaciones básicas, dice el autor, se perfilan "estilos" muy diversos y especificos que se manifiestan en todos los niveles de la vida social. Pinto define "estilo de desarrollo" como el modo en que dentro de un determinado sistema se organizan y asignan los recursos humanos y materiales con el objeto de resolver los interrogantes sobre qué, para quiénes y cómo producir bienes y servicios. Por su parte Graciarena" dice que un "estilo" es la modalidad concreta y dinámica adoptada por un sistema social en un ámbito definido y en un momento histórico determinado. PISPAL, en su documento sobre líneas prioritarias de investigación para la III fase, se ocupa de la definición y de las diversas dimensiones contenidas en los "estilos de desarro1lo". Esas notas de PISPAL apuntan a la tarea mencionada de elaborar adecuadamente el concepto, anotar las diferentes instancias que componen el

${ }_{16}$ Pinto, A., Notas sobre los Estilos de Desarrollo en América Latina, en Revista de la CEPAL, primer semestre, 1976, Santiago de Chile.

"Graciarena, J., Poder y Estilos de Desarrollo. Una Perspectiva Heterodoxa, Revista de la CE$P A L$, primer semestre, 1976, Santiago de Chile. 
mismo y señalar las diferentes acciones y políticas que ponen en funcionamiento y posibilitan cambios de un estilo de desarrollo.

Sin perjuicio de esa tarea teórica que debe continuarse, para los fines inmediatos de su utilización en la investigación empirica debiera pensarse en la selección de algunos indicadores claves que permitan la caracterización de un determinado estilo de desarrollo, su diferenciación de otros estilos y las consecuencias de esas características sobre el fenómeno en estudio. Algunos de estos indicadores serian:

i) características del mercado de trabajo o posibilidades de ofrecer empleo productivo a la población en edad de trabajar, para lo cual interesa conocer: la tasa de participación global y por sexo, para el total del país, regiones y sectores productivos; condición de actividad de la población en edad de trabajar; proporciones de la PEA por rama de actividad, categoría ocupacional y grupos de ocupación; relación entre producto y empleo por rama de actividad y por estratos tecnológicos;

ii) características del mercado de consumo o posibilidades de obtener bienes del mercado, para lo cual interesa conocer: grado de diversificación de los bienes producidos: proporción de los diferentes bienes producidos que son consumidos por los diferentes estratos de ingreso; distribución del ingreso, niveles de salarios;

iii) características de la acción del Estado para mejorar el acceso de la población al mercado de trabajo y al consumo, para lo cual interesa conocer: acceso a los servicios y a la seguridad social para el conjunto de la población, por regiones y áreas de residencia; posibilidad de capacitación de recursos humanos para acceder a empleos más calificados; énfasis en valores de movilidad social y estímulos a logros por roles adquiridos frente roles adscritos; organización de los asentamientos humanos que permitan la real accesibilidad de los servicios sociales al conjunto de la población

iv) condiciones de existencia y posibilidades reales de movilidad social ascendente para la población, según regiones y áreas de residencia.

d) Elección de la unidad de análisis para el estudio de las estrategias de supervivencia. Tanto la definición recogida en la convocatoria de PISPAL, como la generalidad de los trabajos sobre el tema, se refieren a la unidad familiar como organizadora y ejecutora de dichas estrategias. Sin embargo, muchas de las investigaciones empíricas muestran que además de los miembros familiares, frecuentemente existen actores extrafamiliares como allegados, compadres, núcleos con similar situación de deprivación, etc. ${ }^{18}$ que hacen parte, o al menos contribuyen con esas estrategias.

${ }_{18}$ Para los hallazgos de Duque y Pastrana ver nota 2, p. 3. El papel de los compadres y las relaciones de vecindad se muestran en el trabajo de Lomnitz, citado. Respecto a situaciones de núcleos poblacionales con similar situación de deprivación, puede tomarse como ejemplo el caso de los "minifundios informales" estudiados por Maffei, E.; Cambios estructurales en el sector reformado de la agricultura en Chile, su efecto en la demanda de fuerza de trabajo campesina y las migraciones rurales: 1964-1978. GEA, Santiago de Chile, 1980, p. 152. 
Esto debe ser más importante aún en el caso de comunidades étnicas con predominio de pautas culturales muy diferentes, como pueden ser los grupos indígenas. Incluso la definición de familia puede basarse en criterios muy diferentes en esta y en otras culturas. Todo esto nos lleva a pensar que en el nivel de la conceptualización de las "estrategias de supervivencia" no deben hacerse restricciones teóricas que limiten a priori el referente de este concepto a las unidades familiares. En el nivel de las decisiones metodológicas, necesarias para el trabajo de investigación, nos parece adecuado partir con la hipótesis de trabajo de que son las unidades familiares las que ejecutarán aquellas estrategias; pero debe extremarse el requisito siempre presente en la investigación científica, que obliga a estar predispuesto favorablemente a abandonar esa hipótesis si la realidad social no se ajusta bien a nuestros preconceptos.

Entre la posibilidad de que la unidad familiar sea la que organiza y ejecuta la estrategia de supervivencia y la posibilidad de que no lo sea, existirá seguramente un abanico de posibilidades muy rico en hallazgos científicos, que irá desde describir diferentes formas de organización $-\mathrm{y}$ de definición - familiares, hasta variadas formas de interacción entre miembros de la familia y extrafamiliares, sin descartarse la existencia de estrategias individuales y de grupos suprafamiliares.

e) Selección y compatibilización de técnicas de recolección de información. . Entre estas técnicas, corrientes en las diversas disciplinas sociales, se encontrarán diferencias en cuanto al tipo de información que más adecuadamente recogen, así como en cuanto a su cobertura y profundidad. No hay duda de que la técnica de observación participante aparece casi imprescindible si se quiere aprehender un fenómeno social tan complejo y con tantas redes de interacción no registradas en estadísticas de ningún tipo. Por otro lado, los datos censales y de encuestas nacionales de hogares, con todas sus deficiencias actuales, se han mostrado como instrumentos insustituibles cuando se quieren conocer características poblacionales de la sociedad global, superando los estudios de casos que son importantes pero adolecen de limitaciones conocidas.

La observación participante aprehende más adecuadamente el fenómeno pero se limita en cuanto a cobertura; los censos y encuestas de hogares tienen buena cobertura pero difícilmente aprehenden adecuadamente lo que nos interesa conocer. Por cierto, existen antecedentes de integración de diversas técnicas de recolección o uso de información en un mismo proceso de investigación. Una de las tantas formas posibles podria ser la identificación de los grupos condenados por el estilo de desarrollo nacional a ensayar estrategias de superviviencia, utilizando para ello información de censos, encuestas de hogares y otros; para luego, a partir de esa identificación, seleccionar núcleos reducidos representativos de diferentes situaciones, a los cuales se estudiaria en profundidad por medio de técnicas adecuadas, por ejemplo, observación participante, entrevistas en profundidad, etc. 